INPLASY

PROTOCOL

To cite: Li et al. Acupuncture combined with Tongxieyaofang for diarrhea-type irritable bowel syndrome: a protocol for meta-analysis. Inplasy protocol 2020100072. doi: 10.37766/inplasy2020.10.0072

Received: 20 October 2020

Published: 20 October 2020

Corresponding author: Yilin Li

359728736@qq.com

Author Affiliation:

Hospital of Chengdu

University of Traditional

Chinese Medicine

Support: NSFC.

Review Stage at time of this submission: The review has not yet started.

Conflicts of interest: None.

\section{Acupuncture combined with Tongxieyaofang for diarrhea-type irritable bowel syndrome: a protocol for meta-analysis}

\author{
Li, Y1; Yao, C2; Lei, R³ Xie, F4; Xiong, Q5; Luo, L6; Feng, P7.
}

Review question / Objective: The aim of this meta-analysis of randomized controlled trails is to evaluate the efficacy of acupuncture combined with Tongxieyaofang for diarrhea-type irritable bowel syndrome.

Condition being studied: Irritable bowel syndrome (IBS) is a chronic functional bowel disorder characterised by abdominal pain and altered bowel function. The altered bowel function defines the IBS subtypes, of which principally manifesting with diarrhoea is classified as diarrhoea-predominant IBS (IBS-D). IBS-D is highly prevalent, and it causes low work productivity and low quality of life. Current options are limited for the treatment of IBS-D. Several treatments are proposed, but they all have some disadvantages that limit their use in clinical practice. Tong-Xie-Yao-Fang (TXYF) is a Chinese herbal formula for the management of chronic diarrhoea accompanied by abdominal pain; it has been used in China for nearly 600 years. Animal studies showed its efficacy in relieving smooth muscle contraction and decreasing visceral hypersensitivity, and the efficacy may be related to the regulation of 5-hydroxytryptamine and substance $P$ in colonic tissues. Clinical trials showed inconsistent results. The etiology of irritable bowel syndrome remains unclear. Lucky, acupuncture and Chinese medicine have better therapeutic effect on irritable bowel syndrome.

INPLASY registration number: This protocol was registered with the International Platform of Registered Systematic Review and Meta-Analysis Protocols (INPLASY) on 20 October 2020 and was last updated on 20 October 2020 (registration number INPLASY2020100072).

\section{INTRODUCTION}

Review question / Objective: The aim of this meta-analysis of randomized controlled trails is to evaluate the efficacy of acupuncture combined with Tongxieyaofang for diarrhea-type irritable bowel syndrome. 
Condition being studied: Irritable bowel syndrome (IBS) is a chronic functional bowel disorder characterised by abdominal pain and altered bowel function. The altered bowel function defines the IBS subtypes, of which principally manifesting with diarrhoea is classified as diarrhoeapredominant IBS (IBS-D). IBS-D is highly prevalent, and it causes low work productivity and low quality of life. Current options are limited for the treatment of IBSD. Several treatments are proposed, but they all have some disadvantages that limit their use in clinical practice. Tong-Xie-YaoFang (TXYF) is a Chinese herbal formula for the management of chronic diarrhoea accompanied by abdominal pain; it has been used in China for nearly 600 years. Animal studies showed its efficacy in relieving smooth muscle contraction and decreasing visceral hypersensitivity, and the efficacy may be related to the regulation of 5-hydroxytryptamine and substance $P$ in colonic tissues. Clinical trials showed inconsistent results. The etiology of irritable bowel syndrome remains unclear. Lucky, acupuncture and Chinese medicine have better therapeutic effect on irritable bowel syndrome.

\section{METHODS}

Participant or population: Patients who are diagnosed with IBS according to Manning or Rome I, II, III or IV criteria will be included in the analysis, regardless of their age, gender, ethnicity, or background. Participants need to be able to participate in acupuncture combined with Tongxieyaofang treatment to be eligible for inclusion.

Intervention: The types of interventions comprised trials that compared acupuncture combined with Tongxieyaofang for the treatment of patients with IBS-D.

Comparator: The control group received conventional treatment of Western medicine, including the use of PPI, gastrointestinal motility drugs, or a combination of both.
Study designs to be included: Randomized controlled trials (RCTs) that investigated the effect of acupuncture combined with Tongxieyaofang for the treatment of diarrhea-type irritable bowel syndrome will be included. No language limitation exists.

Eligibility criteria: Records from database searches will be managed by EndNoteX7 software. The titles and abstracts of each record retrieved will be checked by 2 independent authors according to eligibility criteria. The full texts of potentially relevant studies will be retrieved for further assessment. Disagreements will be resolved by discussion or consultation of a 3rd author. A data spreadsheet will be created using Microsoft Excel 2007 to collect relevant information and data.The information, including author, year of publication, sample size, intervention, and outcome will be extracted from each study and entered into the spreadsheet.

Information sources: We will search the following Electronic databases: PubMed, the Cochrane Library, Embase, Web of Science, the China National Knowledge Infrastructure, Wanfang Database, Chinese Science and Technology Periodical Database, and Chinese Biomedical Literature Database.

Main outcome(s): The main outcome include effectiveness, basic recovery, marked effect, improvement, remission rate, relapse rate, clinical absolute score, and relative score.

Additional outcome(s): Secondary outcome indicators: including any related adverse reactions, the concentration of serotonin in Serum.

Quality assessment / Risk of bias analysis: The quality of evidence of outcomes will be assessed by two authors according to the "Bias Risk Assessment"tool recommended by Cochrane Collaboration Network (Version5.1.0) which include selection bias (method of randomization and allocation concealment), information bias (masking of outcome adjudicators), and bias in the analysis (intention to treat 
analysis and completeness of follow-up). The strength of the body of evidence will be graded into 3 levels, "High Risk", "Low Risk", "Unclear". Disagreement which existed between 2 author's results will be solved by discussion and settled through consultation with the third party . Bias risk assess figure will be drawn by RevMan software5.3.

Strategy of data synthesis: When meta analysis is available, RevManV5.3 will be applied to analyze data. Data will use a random effects model with $95 \% \mathrm{Cls}$ as substantial heterogeneity is expected among included studies. The heterogeneity will be analyzed before meta-analysis, we will use 12 statistics to assess heterogeneity across included studies. If $\mathrm{P}$ value $<.10$ and/or $1250 \%$, we will use random effects model to make metaanalysis, and sensitivity analysis or subgroup analysis is needed to identify the sources of heterogeneity among the included studies. And the 2-side $P$ value $<.05$ in Z-test will be considered as statistically significant.

Subgroup analysis: Subgroup analysis will also be employed to explore the possible causes of heterogeneity. Subgroup analysis will be based on possible factors that may lead to heterogeneity, such as intervention measures, control measures, length of treatment or quality of articles, etc.If quantitative synthesis not appropriate, we will conduct a narrative synthesis.

Sensibility analysis: The quality of evidence of outcomes will be assessed by two authors according to the"Bias Risk Assessment"tool recommended by Cochrane Collaboration Network (Version5.1.0) which include selection bias (method of randomization and allocation concealment), information bias (masking of outcome adjudicators), and bias in the analysis (intention to treat analysis and completeness of follow-up). The strength of the body of evidence will be graded into 3 levels, "High Risk",“Low Risk”,"Unclear”. Disagreement which existed between 2 author's results will be solved by discussion and settled through consultation with the third party . Bias risk assess figure will be drawn by RevMan software5.3.

Language: The language is limited to Chinese and English.

Country(ies) involved: China.

Keywords: Irritable bowel syndrome, network meta-analysis, protocol, acupuncture, Tongxieyaofang.

Contributions of each author:

Author 1 - Yilin Li - The author drafted the manuscript.

Author 2 - Chenhjiao Yao - The author provided statistical expertise.

Author 3 - Rong Lei - The author contributed to the development of the selection criteria, and the risk of bias assessment strategy.

Author 4 - Fengjiao Xie - The author read, provided feedback and approved the final manuscript.

Author 5 - Qin Xiong - The author read, provided feedback and approved the final manuscript.

Author 6 - Lihong Luo - The author read, provided feedback and approved the final manuscript.

Author 7 - Peimin Feng - The author provide scientific research ideas and guidance. 